\title{
A Mathematical Study of an Influenza Model with Vaccination
}

\author{
Siwaphorn Kanchanarat and Settapat Chinviriyasit
}

\begin{abstract}
Influenza is the serious disease caused by an influenza virus. This disease is spreading widely that difficult to control. Vaccination is a commonly method for protect disease. In this study, thus, SVEIR (susceptible-vaccinated-exposed-infectious-recovered) model is used to predict the number of infected and the duration of an outbreak when it occurs. Using next generation method, it is shown that the disease can be eradicated from the population if the vaccination coverage level exceeds a certain threshold value. It is also shown that the disease will persist within the population if the coverage level of vaccination is below this critical value. These results are verified by numerical simulations.
\end{abstract}

Index Terms - SVEIR epidemic model, stability, disease free equilibrium point, basic reproductive number.

\section{INTRODUCTION}

Influenza is an infectious disease caused by influenza virus and transmitted among humans. It can be transmitted via direct contact, indirect contact through contaminated objects, as well as droplets and aerosols resulting from coughs or sneezes. Despite vaccination and other prevention and control efforts, influenza mortality, morbidity and economic burden remains significant. Worldwide and annually, influenza infection is between $5-15 \%$ of the population, and mortality can reach up to 500,000 [1]. In fact, vaccination can only offer a tempolary immunity to the disease. Thus, once a vaccine wanes from the body of the vaccinated person, the person becomes susceptible to the disease again. Hence, it is necessary to develop a framework that could predict the optimal level of vaccine coverage needed to prevent the spread of an endemic.

Mathematical modeling of the spread of influenza can play an important role in providing deeper insights into the aforementioned transmission dynamics of diseases and to evaluate different control strategies (see, for instance, [2]-[7], [8]. Samsuzzoha et al. [6] analyzed an influenza epidemic model based on the sensitivity indices of the basic reproductive number and the endemic point of equilibrium to the parameters, The result showed that the basic reproduction number is the most sensitive to the transmission rate of the disease. However, this work has not been done in terms of the mathematical modeling. The aim of this paper is to provide a

Manuscript received November 13, 2013; revised January 14, 2014. This work was supported in part by the National Centre of Exellence in Mathematics under the Commission on Higher Educational, Ministry of Education, Thailand

The authors are with the Department of Mathematics at King Mongkut's University of Technology Thonburi, Bangkok, 10140 Thailand (e-mail: siwaphonkp@gmail.com, settapat.chi@kmutt.ac.th). qualitative study of the influenza model with vaccination proposed by Samsuzzoha et al. [6] for predicting the optimal vaccination coverage needed to ensure that the disease does not spread and to determine the important of the epidemic model parameters.

The model monitors the dynamics of the populations of susceptible, $S$, vaccinated, $V$, exposed, $E$, infective, $I$ and recovered, $R$, which is represented by the following system:

$$
\begin{aligned}
& \frac{d S}{d t}=-\beta \beta_{E} \frac{E S}{N}-\beta \beta_{I} \frac{I S}{N}-\phi S-\mu S+\delta R+\theta V+r N, \\
& \frac{d V}{d t}=-\beta \beta_{E} \beta_{V} \frac{E V}{N}-\beta \beta_{I} \beta_{V} \frac{I V}{N}-\mu V-\theta V+\phi S, \\
& \frac{d E}{d t}=\beta \beta_{E} \frac{E S}{S}+\beta \beta_{I} \frac{I S}{S}+\beta \beta_{E} \beta_{V} \frac{E V}{N}+\beta \beta_{I} \beta_{V} \frac{I V}{N}-(\mu+\kappa+\sigma) E, \\
& \frac{d I}{d t}=\sigma E-(\mu+\alpha+\gamma) I, \\
& \frac{d R}{d t}=\kappa E+\gamma I-\mu R-\delta R .
\end{aligned}
$$

where $N=S+V+E+I+R$ be the total population. The biological meaning of the all parameters in (1) and chosen values of these parameters are given in the Table I.

\section{ANALYSIS OF THE MODEL}

\section{A. Dimensionless}

To reduce the model (1) in terms of the dimensionless proportions of susceptible, vaccinated, exposed, infectious and recovered populations, let

$$
s=\frac{S}{N}, v=\frac{V}{N}, e=\frac{E}{N}, i=\frac{I}{N}, \quad r_{1}=\frac{R}{N}
$$

After some manipulations and replacing $S$ by $S, v$ by $V, e$ by $E, I$ by $I$ and $r_{1}$ by $R$, the system (1) can writen as

$$
\begin{aligned}
& \frac{d S}{d t}=-\beta \beta_{E} E S-\beta \beta_{I} I S+\alpha I S-\phi S-r S+\delta R+\theta V+r, \\
& \frac{d V}{d t}=-\beta \beta_{E} \beta_{V} E V-\beta \beta_{I} \beta_{V} I V-\alpha I V-\theta V+\phi S, \\
& \frac{d E}{d t}=\beta \beta_{E} E S+\beta \beta_{I} I S+\beta \beta_{E} \beta_{V} E V+\beta \beta_{I} \beta_{V} I V-(r+\kappa+\sigma) E, \\
& \frac{d I}{d t}=\sigma E-(r+\alpha+\gamma) I+\alpha I^{2}, \\
& \frac{d R}{d t}=\kappa E+\gamma I-r R-\delta R+\alpha I R .
\end{aligned}
$$

This allows us to attack (1) by studying the system (2). From biological considerations, we study (2) in the closed set 


$$
T=\left\{(S, V, E, I, R) \in \mathbb{R}_{+}^{5} \mid S+V+E+I+R=1\right\},
$$

where $\mathbb{R}_{+}^{5}$ denotes the non-negative cone and its lower dimensional faces. It can be verified that $T$ is positively invariant with respect to (2). Hence, it is sufficient to consider the dynamics of the flow generated by models (2) in $T$.

TABLE I: MODEL PARAMETERS [5]

\begin{tabular}{lll}
\hline \hline Parameters & Description & Value \\
\hline$\beta$ & Contact rate & 0.514 \\
$\beta_{E}$ & $\begin{array}{l}\text { Ability to cause infection by exposed } \\
\text { individuals }\end{array}$ & 0.250 \\
$\beta_{I}$ & $\begin{array}{l}\text { Ability to cause infection by infectious } \\
\text { individuals }\end{array}$ & 1.000 \\
$\beta_{V}$ & Ability to cause infection by vaccination & 0.1 \\
$\sigma$ & individuals & 0.500 \\
$\gamma$ & Rate of latency & 0.200 \\
$\delta$ & Rate of clinically ill & $1 / 365$ \\
$\mu$ & Rate of duration of immunity loss & $5.5 \times 10^{-8}$ \\
$r$ & Natural mortality rate & $7.140 \times 10^{-5}$ \\
$\kappa$ & Birth rate & $1.857 \times 10^{-4}$ \\
$\alpha$ & Recovery rate of latents & $9.3 \times 10^{-6}$ \\
$\theta$ & Flu induced mortality rate & $1 / 365$ \\
$\phi$ & Rate of susceptible & Variable \\
\hline \hline
\end{tabular}

\section{B. Disease-Free Equilibrium (DFE)}

The model (2) has a DFE, obtained by setting all derivatives in the system (2) to zero, given by

$$
P^{0}=\left(S^{0}, V^{0}, E^{0}, I^{0}, R^{0}\right)=\left(\frac{r+\theta}{r+\theta+\phi}, \frac{\phi}{r+\theta+\phi}, 0,0,0\right)
$$

The linear stability of $P^{0}$ is established using the next generation operator method [7] as follows. The next generation matrices, $F$ and $V$, are given by

$$
F=\left[\begin{array}{cc}
\beta \beta_{E} & \beta \beta_{I} \\
0 & 0
\end{array}\right] \text { and } V=\left[\begin{array}{cc}
\kappa+\sigma+r & 0 \\
-\sigma & \alpha+\gamma+r
\end{array}\right]
$$

respectively.

Letting $R_{v a c}=\rho\left(F V^{-1}\right)$, it follows that

$$
R_{v a c}=R_{0}\left(\frac{r+\theta+\beta_{V} \phi}{r+\theta+\phi}\right)
$$

where $R_{0}=\frac{\beta\left(r \beta_{E}+\alpha \beta_{E}+\gamma \beta_{E}+\sigma \beta_{I}\right)}{(r+\alpha+\gamma)(r+\kappa+\sigma)}$ is the basic reproductive number of infection for the vaccination-free model $(\phi=0)$.

According the next generation operator method [9], the local stability of the DFE, $P^{0}$, is based on whether or not $\rho\left(F V^{-1}\right)<1$, then all eigenvalues of the linearized version of the system (2) have negative real parts, so that the DFE is locally asymptotically stable (LAS). For $\rho\left(F V^{-1}\right)>1$, at least one of the eigenvalues has a positive real part, thus, the DFE is unstable in this case. Consequently, the following is established by using Theorem 2 of [9].

Lemma 1: The disease free equilibrium, $P^{0}$, of the system (1) is locally asymptotically stable if $R_{v a c}<1$ and unstable if $R_{v a c}>1$.

The quantity $R_{V A C}$ is the basic reproduction number of the model (1). It measures the average number of secondary infection cases produced by an infectious individual during its entire infectious period. The local stability result in Lemma 1 implies that for $R_{V A C}<1$, the total number of infectious individual in the population can be reduced to zero. This verifies in Fig. 1. Therefore, in the event of an epidemic, the theoretical determination of conditions that can make $R_{V A C}$ less than unity is of great public health interest.

\section{Endemic Equilibrium}

Let $P^{*}=\left(S^{*}, V^{*}, E^{*}, I^{*}, R^{*}\right)$ be endemic equilibrium of the model (2). Since the endemic equilibrium of the system (2) can not be written in the closed form, then, for convenient, the endemic equilibrium (that is, there exists at least one of infected components which is non-zero) is obtained by solving the model (1) with the assumption $\mu=r$ as in the following steps. Let

$$
G^{*}=\beta \beta_{E} \frac{E^{*}}{N^{*}}+\beta \beta_{I} \frac{I^{*}}{N^{*}}
$$

represents the force of infection at steady state. By setting all derivatives in the model (1) equal to zero and solving the state variables of the model in terms of $G^{*}$ yield

$$
\begin{aligned}
S^{*} & =\frac{\delta R^{*}+\theta V^{*}+r N}{G+\phi+r}, \\
V^{*} & =\frac{\phi S^{*}}{G \beta_{V}+r+\theta}, \\
E^{*} & =\frac{G S^{*}+G \beta_{V} V^{*}}{r+\kappa+\sigma}, \\
I^{*} & =\frac{\sigma E^{*}}{r+\alpha+\gamma}, \\
R^{*} & =\frac{\kappa E^{*}+\gamma I^{*}}{r+\delta} .
\end{aligned}
$$

Substituting (5) into the expression for $G^{*}$ in (5) and simplifying, gives the equation:

$$
G^{*}\left(a_{0} G^{* 2}+b_{0} G^{*}+c_{0}\right)=0
$$

where

$$
\begin{gathered}
a_{0}=\beta_{V}\left[e_{2}(\mu+\delta+\kappa)+\sigma(\mu+\delta+\gamma)\right], \\
b_{0}=(\mu+\delta)\left\{\beta_{V}\left(e_{1} e_{2}-e_{2} \beta_{1}-\sigma \beta_{2}\right)+\left(e_{2}+\sigma\right)\right\}+e_{2} \kappa+\sigma \gamma \\
c_{0}=e_{1} e_{2}(\mu+\delta)(\mu+\theta+\phi)\left[1-R_{V A C}\right] .
\end{gathered}
$$

where

$$
\begin{aligned}
& \beta_{1}=\beta \beta_{E}, \beta_{2}=\beta \beta_{I} \\
& e_{1}=\mu+\kappa+\sigma, e_{2}=\mu+\alpha+\gamma
\end{aligned}
$$


Clearly, $G^{*}=0 ;$ corresponds to the diseases from equilibrium $P^{0}$ given in (3).

For $G^{*} \neq 0$; the positive equilibrium of the model (1) can be obtained by solving

$$
a_{0} G^{* 2}+b_{0} G^{*}+c_{0}=0
$$

for $G^{*}$ and substituting the result into (5).

The analysis of (8) is Theorem 1 .

Theorem 1: The model (2) has an unique endemic equilibrium, $P^{*}$, whenever $R_{V A C}>1$.

\section{The Optimal Vaccine Coverage Level}

The epidemiological implication of Lemma 1 is that if model parameters can be selected (either via vaccination or other control measures) such that the basic reproductive number, $R_{V A C}$ is less than unity, then the disease will be eradicated from the community. The effect of vaccinated rate, $\phi$, on $\quad R_{V A C}$ is investigated by using sensitivity analysis (i.e., differentiating $R_{V A C}$ partially with respect to control parameter $\phi$ ). It is found that

$$
\frac{\partial R_{V A C}}{\partial \phi}=-\frac{\left(1-\beta_{V}\right)(r+\theta)}{(r+\theta+\phi)^{2}} R_{0}<0
$$

from which it follows that $R_{V A C}$ is decreasing function of $\phi$. It is clear that vaccination is critically important in making $R_{V A C}$ less than unity. This implies that vaccination to susceptible populations will reduce number of infections down.

From the definition of $R_{V A C}$ in (3), it can be seen that if

$$
\phi_{C}=\frac{(r+\theta)\left(R_{0}-1\right)}{1-\beta_{V} R_{0}}
$$

then $R_{V A C}=1$. Since $R_{V A C}$ is a decreasing function of $\phi$, it follows that if $\phi>\phi_{C}$ then $R_{V A C}<1$. Thus, the condition for disease eradication is satisfied if $\phi>\phi_{C}$ and $\phi_{C}$ is called the optimal vaccine coverage level needed for disease eradication.

\section{E. The Effect of the Rate of Recovery $(\gamma)$ Latency $(k)$ and Vaccination-Induced Immunity Loss $(\theta)$ on $R_{V A C}$}

By differentiating $R_{V A C}$ partially with respect to control parameters $\gamma, \kappa$ and $\theta$, respectively, yield

$$
\begin{gathered}
\frac{\partial R_{V A C}}{\partial \gamma}=-\frac{\left(\sigma \beta_{I}\right)\left(r+\theta+\beta_{V} \phi\right)}{(r+\theta+\phi)(r+\kappa+\sigma)(r+\alpha+\gamma)^{2}}<0, \\
\frac{\partial R_{V A C}}{\partial \kappa}=-\frac{\beta\left(r \beta_{E}+\alpha \beta_{E}+\gamma \beta_{E}+\sigma \beta_{I}\right)\left(r+\theta+\beta_{V} \phi\right)}{(r+\alpha+\gamma)(r+\theta+\phi)(r+\kappa+\sigma)^{2}}<0, \\
\frac{\partial R_{v a c}}{\partial \theta}=\frac{\beta \phi\left(1-\beta_{V}\right)\left(r \beta_{E}+\alpha \beta_{E}+\gamma \beta_{E}+\sigma \beta_{I}\right)}{(r+\alpha+\gamma)(r+\kappa+\sigma)(r+\theta+\phi)^{2}}>0 .
\end{gathered}
$$

Clearly, $R_{V A C}$ is decreasing function of $\gamma$ and $\kappa$, see (11) and (12), respectively. Whereas $R_{V A C}$ is increasing function of $\theta$, see (13).

\section{NUMERICAL SIMULATIONS}

To observe the dynamic behavior of the model, the system (1) is integrated numerically by using fourth order Rungge-Kutta method with the parameter values in Table I , $\mathrm{N}=1,000,000$ and various value of $\phi$. The initial conditions [4] are

$$
S(0)=0.799, V(0)=0.197, E(0)=0, I(0)=0.004, R(0)=0 .
$$

With parametervalues in Table I, the threshold vaccination coverage or critical vaccination parameter is $\phi_{c}=0.00715$. Table II depicts the variables of the model at steady-state as a function of $\phi$ and $R_{V A C}$. It is clear from this table that when the vaccination coverage level $(\phi)$ increases, the value of $R_{V A C}$ decreases. The result verify that the endemic equilibrium, $P^{*}=\left(S^{*}, V^{*}, E^{*}, I^{*}, R^{*}\right)$, (that is the number of exposed $\left(E^{*}\right)$ and infectious ( $\left.I^{*}\right)$ individuals are not zero) is stable if the vaccination coverage level $(\phi)$ is below the threshold $\phi_{C}$. Thus, the disease will persist in the population since $R_{V A C}$ is greater than unity. The profiles of infected populations for $\phi=0,0.003,0.005$. are depicted in Fig. 2. The result show, as $\phi$ increases, the number of infectious individual decreases and the duration of outbreak is delayed before convergence to the corresponding endemic equilibrium $P^{*}$ as shown in Table II. However, when $\phi$ is increased to values greater than $\phi_{C}$, such as $\phi=0.0072$, Table II confirms that the disease-free equilibrium $\left(P^{0}\right)$ is stable (since $R_{V A C}$ is less than unity in this case) and the infected population (the sum of exposed and infected individuals) vanishes in time. This leads to the eradication of the disease from the community. These simulation results are in line with Lemma1 in Section II.
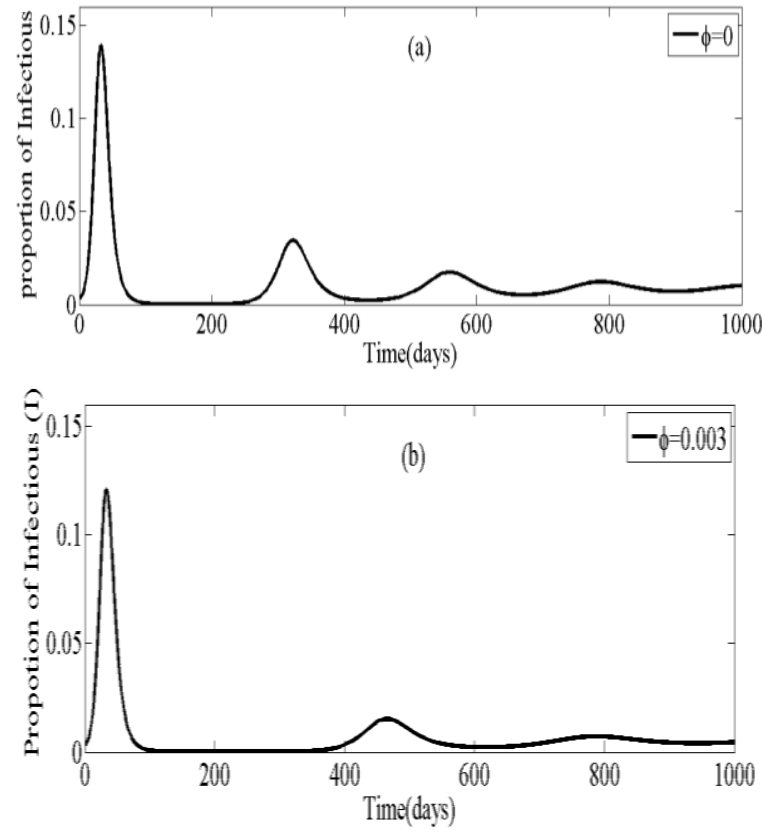


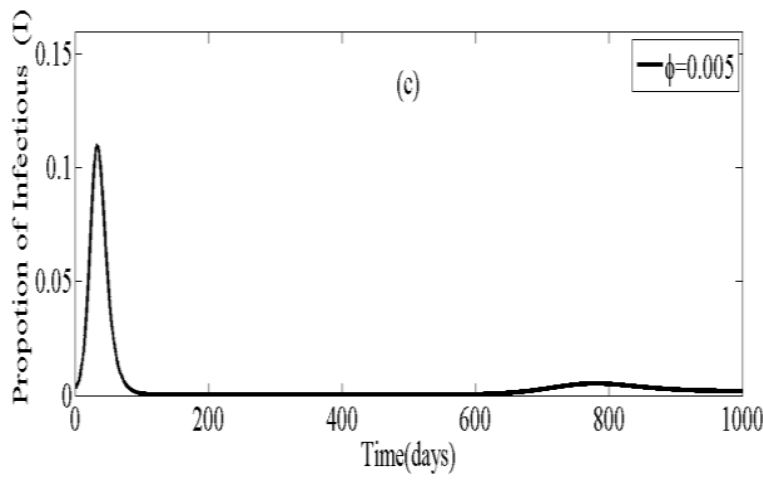

Fig. 1. Profile of the propotion of infectious individuals using $\phi=0,0.003,0.005$.

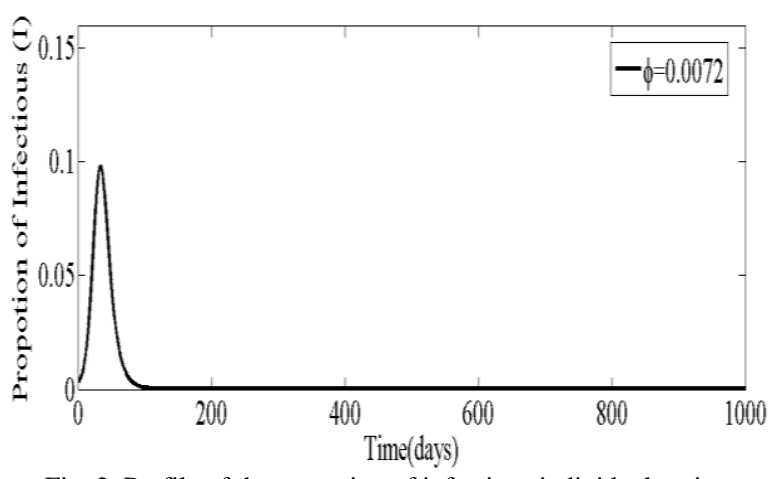

Fig. 2. Profile of the propotion of infectious individuals using $\phi=0.0072$.

The effect of the recovery rate of latent, recovery rate of infectious and vaccination-induced immunity loss rate are investigated using the parameter values in Table I, $\phi=0.001$. and vary the parameters $\kappa, \gamma$ and $\theta$, respectively. The results are tabulated in Table III-Table V. Table III and Table IV show that the number of infectious individuals decrease as $\kappa$ and $\gamma$ increase. Table $\mathrm{V}$ also show that increasing the duration of the loss of immunity induced by vaccination increase the number of of infectious individuals because it reduces the threshold vaccination coverage $\left(\phi_{c}\right)$ which is critically important for the success of public health strategies for controlling an epidemic.

TABLE II: EFFECT OF VACCINATION COVERAGE ( $\phi)$ ON $R_{V A C}$ AND

$$
S^{*}, V^{*}, E^{*}, I^{*}, R^{*} \text { AT STEAdy StATE }
$$

\begin{tabular}{lllllll}
\hline \hline$\phi$ & $R_{v a c}$ & $S^{*}$ & $V^{*}$ & $E^{*}$ & $I^{*}$ & $R^{*}$ \\
\hline 0 & 2.82 & 354,045 & 0 & 3,562 & 8,900 & 633,493 \\
0.003 & 1.51 & 322,759 & 312,855 & 2,009 & 5,021 & 357,356 \\
0.005 & 1.19 & 302,812 & 512,349 & 1,019 & 2,546 & 181,274 \\
0.007 & 1.01 & 283,657 & 703,902 & 68 & 171 & 12,202 \\
0.0071 & 1.00 & 282,718 & 713,261 & 22 & 55 & 3,944 \\
0.0072 & 0.99 & 280,800 & 719,200 & 0 & 0 & 0 \\
\hline
\end{tabular}

TABLE III: EFFECT OF RECOVERY RATE OF LATENTS $(\kappa)$ ON $R_{V A C}$ And $S^{*}, V^{*}, E^{*}, I^{*}, R^{*}$ at Steady State

\begin{tabular}{lccclcl}
\hline \hline \multicolumn{1}{c}{$\kappa$} & $R_{v a c}$ & $S^{*}$ & \multicolumn{1}{c}{$V^{*}$} & $E^{*}$ & \multicolumn{1}{c}{$I^{*}$} & \multicolumn{1}{c}{$R^{*}$} \\
\hline 0 & 2.15 & 343,316 & 105,952 & 3,037 & 7,591 & 540,104 \\
0.1 & 1.79 & 411,447 & 132,370 & 2,103 & 5,256 & 448,824 \\
0.3 & 1.34 & 547,613 & 186,154 & 924 & 2,310 & 262,999 \\
0.5 & 1.07 & 683,706 & 240,667 & 210 & 526 & 74,891 \\
0.55 & 1.02 & 717,723 & 254,368 & 74 & 185 & 27,650 \\
0.6 & 0.98 & 737,610 & 262,390 & 0 & 0 & 0 \\
\hline
\end{tabular}

TABLE IV: EFFECT OF RECOVERY RATE OF INFECTIOUS $(\gamma)$ ON $R_{V A C}$ AND $S^{*}, V^{*}, E^{*}, I^{*}, R^{*}$ AT STEADY STATE

\begin{tabular}{lllllll}
\hline \hline$\gamma$ & $R_{v a c}$ & $S^{*}$ & $V^{*}$ & $E^{*}$ & $I^{*}$ & $R^{*}$ \\
\hline 0.1 & 4.11 & 180,939 & 45,862 & 4,206 & 21,017 & 747,976 \\
0.2 & 2.15 & 343,444 & 106,001 & 3,035 & 7,586 & 539,934 \\
0.3 & 1.50 & 491,523 & 163,890 & 1,908 & 3,180 & 339,499 \\
0.4 & 1.17 & 627,159 & 217,960 & 859 & 1,074 & 152,948 \\
0.45 & 1.06 & 690,806 & 243,529 & 364 & 405 & 64,896 \\
0.5 & 0.98 & 737,610 & 262,390 & 0 & 0 & 0 \\
\hline
\end{tabular}

TABLE V: EFFECT OF RATE OF VACCINATION-INDUCED IMMUNITY $\operatorname{Loss}(\theta)$ ON $R_{V A C}$ AND $S^{*}, V^{*}, E^{*}, I^{*}, R^{*}$ AT StEADY State

\begin{tabular}{lccclll}
\hline \hline$\theta$ & $R_{\text {vac }}$ & $S^{*}$ & $V^{*}$ & $E^{*}$ & $I^{*}$ & $R^{*}$ \\
\hline 0.0001 & 0.65 & 146,320 & 853,680 & 0 & 0 & 0 \\
0.0002 & 0.82 & 213,465 & 786,535 & 0 & 0 & 0 \\
0.0003 & 0.97 & 270,818 & 729,182 & 0 & 0 & 0 \\
0.00035 & 1.03 & 297,987 & 560,569 & 779 & 1,948 & 138,717 \\
0.0004 & 1.10 & 306,101 & 479,436 & 1,182 & 2,955 & 210,326 \\
\hline
\end{tabular}

\section{CONCLUSION}

An influenza model with vaccination presented in [5] is analyzed to gain insight into their dynamical features and used to monitor transmission dynamics in a population. The study shows the following:

1) Detailed local stability analysis of the model reveals that the disease-free equilibrium is locally asymptotically stable when $R_{V A C}<1$ and unstable when $R_{V A C}>1$.

2) The vaccination coverage level, $\phi_{C}$, is defined and is given by

$$
\phi_{C}=\frac{(r+\theta)\left(R_{0}-1\right)}{1-\beta_{V} R_{0}}
$$

where $R_{0}$ is the reproductive number of infection for the vaccination-free model ( $\phi=0)$.

3) The reproductive number $R_{V A C}$ is less than one provided the vaccination coverage level exceeds a certain threshold $\phi_{C}$. This result predicts in Table II. Meanwhile, the endemic equilibrium is stable if the vaccination coverage level $(\phi)$ is less than $\phi_{C}$, see Table II and Fig. 2. Meanwhile, the endemic equilibrium is stable if the vaccination coverage level $(\phi)$ is less than $\phi_{C}$, see Table II and Fig. 1.

4) The relative importance parameters in the transmission are tabulated in Tables I-V. These results $\phi, \theta, \kappa, \gamma$ are the sensitive parameters for $S^{*}, V^{*}, E^{*}, I^{*}, R^{*}$. The results also show that the use of vaccines that offer life-long protection is a crucial public health objective for disease control or eradication. This is especially critical in countries where finances play a critical role in the number of people who receive vaccines.

\section{ACKNOWLEDGMENT}

The work was supported by the National Centre of Exellence in Mathematics under the Commission on Higher Educational, Ministry of Education, Thailand. 


\section{REFERENCES}

[1] WHO. (2009). Influenza(Sensonal). [Online]. Available: http://www.who.int/mediacentre/factsheets/fs211/en/

[2] B. T. Grenfell and R. M. Anderson, "Pertusis in England and Wales: an investigation of transmission dynamics and control by mass vaccination," Proc.Roy. Soc. London, vol. 236, pp. 213-252, 1989.

[3] D. J. D. Earn, J. Dushoff, and S. A. Levin, "Ecology and evolution of the flu," Trends Ecol, vol. 17, pp. 334-340, 2002.

[4] J. C. Helton, F. J. Davis, and J. D. Johnson, "A comparison of uncertainty and sensitivity analysis results obtained with random and Latin hypercube sampling," Reliab. Eng. Syst Safe, vol. 89. pp. 305-330, 2005.

[5] M. Samsuzzoha, M. Singh, and D. Lucy, "A numerical study on an influenza epidemic model with vaccination and diffusion," Applied Mathematics and Conputation, vol. 219, pp.122-141, 2012.

[6] Md. Samsuzzoha, M. Singh, and D. Lucy, "Uncertainty and sensitivity analysis of the basic reproduction number of a vaccinated epidemic model of influenza," Applied Mathematical Modelling, vol. 37, pp. 903-915, 2013.

[7] R. J. Webby and R. G. Webster, "Are we ready for pandemic influenza?" Science, vol. 302, pp. 1519-1522, 2003.

[8] Y. Deguchi and Y. Tagasugi, "Efficacy of influenza vaccine in the elderly:reduction in risk of mortality and morbidity during an influenza A (H3N2) epidemicfor the elderly in nursing homes," Int. J. Clin. Lab. vol. 30, pp. 1-4, 2000.
[9] P. V. D. Driessche and J. Watmough, "Reproduction Numbers and Sub-Threshold Endemic Equilibria for Compartmental Models of Disease Tramsmission," Mathematical Biosciences, vol. 180, pp. 29-48.

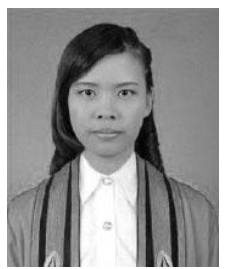

Siwaphorn Kanchanarat was born on 8th December 1989. She was graduated bachelor degree of science (applied computer science) at King Mongkut's University of Technology Thonburi, 2011.

Now, she is studying master degree of science (applied mathematics) in Department of Mathematics at King Mongkut's University of Technology Thonburi.

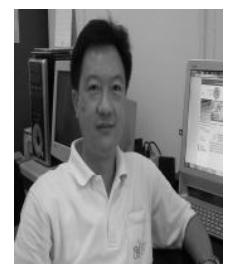

Settapat Chinviriyasit is currently employed as an associated professor and a doctor in mathematics at King Mongkut's University of Technology Thonburi, Thailand.

He obtained his PhD from Brunel University (UK). His interested research fields are as follow: numerical modeling of differential equations by using Finite difference and finite element methods, mathematical modeling in ecology and biology, applied dynamical systems, computational mathematics. 